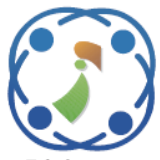

\title{
An Energy Efficient Multicast Route Establishment using AODV with PSO Algorithm and RSA for Secured Transmission
}

\author{
Viyyapu Lokeshwari Vinya ${ }^{1,2 *}$ \\ Gurrala Venkateswara Rao ${ }^{3}$ \\ ${ }^{1}$ Department of Information Technology, Gandhi Institute of Technology and Management University, \\ Visakhapatnam, Andhra Pradesh, India \\ ${ }^{2}$ Department of Computer Science and Engineering, Gayatri Vidya Parshad College of Engineering (Autonomous), \\ Visakhapatnam, Andhra Pradesh, India \\ ${ }^{3}$ Department of Information Technology, GITAM Institute of Technology, \\ Gandhi Institute of Technology and Management University, Visakhapatnam, India \\ * Corresponding author's Email: vinya593@ gmail.com
}

\begin{abstract}
Cognitive Radio (CR) is an emerging technology to solve the issue of scarce spectrum resource utilization. The routing plays a significant role in CR Ad-hoc network and it has two major problems, those are spectrum load balance and energy efficiency. Hence, maintaining the spectrum load balance and energy efficiency becomes a difficult task in CR Ad-hoc Network. In this research, a new Multicast Ad-hoc On-Demand Distance Vector (MAODV) with Particle Swarm Optimization (PSO) algorithm was introduced for selecting best route of less energy consumption in the CR networks. The routing algorithm is implemented with an efficient particle encoding scheme and multi-objective fitness function. Moreover, the data packets are secured by using the RSA algorithm. The optimized lifetime attained from the desired CR ad-hoc networks by using this RSA technique. The multiple scenarios were simulated to compute and analyse the performances in terms of energy consumption, Packet Delivery Ratio (PDR), end to end delay, and throughput. The proposed method named as MAODV-PSO-RSA, which implemented in Network Simulator-2 (NS2). The main objective of the MAODV-PSO-RSA method is to improve the energy consumption during the routing process. The simulation results showed that MAODV-PSO-RSA method had improved 1- $2.2 \%$ of network performance compared to the existing methods such as Bio-Inspired Routing Protocol (BIRP) and Improved Frog Leap Inspired Protocol (IFLIP).
\end{abstract}

Keywords: Cognitive radio, Multicast ad-hoc on-demand distance vector, Particle swarm optimization, Rivest Shamir Adleman, Security.

\section{Introduction}

The CR allows unlicensed secondary users to access spectrum bands, which are temporarily not utilized by licensed Primary Users (PUs) [1-3]. Routing plays a significant role in the CR ad-hoc network, it has two main issues like energy efficiency and spectrum load balance [4,5]. Generally, the routing protocols avoid areas that are highly congested with PUs, leaving only a small fragment of obtainable links used for secondary route construction in CR networks [6]. The energyaware routing protocol makes use of the relative energy metric to select the optimal route, hence it reduces the average total power consumption and maintains the network lifetime [7]. The CR routing protocol used for ad-hoc networks that particularly address the concerns of end-end CR performance over multiple hops and issue of protecting the primary user transmissions from interference with limited knowledge of environment [8]. Routing concept is the most significant problem to be addressed and desires absolute investigation to deal with CR network. In the past years, the routing protocols assumed the presence of the associated path from the transmitter into the receiver. Sometimes this assumption is to be invalid [9]. So, 
an efficient routing protocol requires to find stable routes as well as to confirm very fast data delivery from the transmitter nodes to receiver nodes of the CR network [10]. CR networks gained considerable attentions to ease the contradiction between the scarcity and low utilization of spectrum resources. However, high energy consumption is one of the major challenges encountered in traditional works.

To overcome this problem, this research introduces an efficient routing protocol with PSO algorithm for attaining less energy consumption in the $C R$ network. This proposed MAODV performs the Joint Node Channel Assignment (JNCA) by taking energy into $\mathrm{CR}$ at the network layer. After routing protocol process, the sending data packets are secured by RSA algorithm. The optimized lifetime is obtained from the desired CR network by RSA algorithm. The proposed MAODV-PSO-RSA is experimented widely and results are compared with conventional routing algorithms to prove their superiority in terms of energy consumption, delay, PDR, packet loss. In this research address the following issues:

1. Energy efficient routing with a trade-off between transmission distance and number of data forwards.

2. The CR technology is primarily proposed to tackle spectrum resource usage and security problems, but it's in built properties that encourages more energy consumption creates it a significant aspect of research in field of communication.

This research work is composed as follows; section 2 surveys on existing research works based on routing protocol and security in CR network. In section 3, a brief explanation of a MAODV-PSORSA method is presented for enhancing the performance of CR networks. In the section 4, the comparison of the experimental results of existing and MAODV-PSO- RSA algorithms are presented. The conclusion is made in section 5 .

\section{Literature survey}

This section analyses some significant research approaches suggested by the researchers on routing protocol and security over the CR network. A brief evaluation of some significant contributions of the conventional routing protocol and security methods is presented.

Wang et al. [11] proposed a Joint Channel Selection (JCS) and a routing protocol for the CR network. A joint interaction between channel selection and routing protocol creates switching delay and account availability to select the shortest delay as the best path. JCS method effectively reduced the number of the channel failure by the considering channel's historical information and routing maintenance to adapt location of multichannel CR network. The experimental results showed that the proposed method performed better in terms of the Packet Transmission Delay (PTD) and Data Delivery Rate (DDR). But, the proposed method requires more execution time.

Anamalamudi et al. [12] proposed the AODV routing protocol for $\mathrm{CR}$ access based on the Internet of Things (IoT). In this research, the Hybrid Control Channel (HCC) based on cognitive AODV with directional antennas was proposed to find the channel route from Leaf Nodes Border Router (LBR) to the destination associated within the CR network. The proposed routing protocol was used to transmit the IoT constrained data from IoT border node to $\mathrm{CR}$ destination. The simulation results showed that the data transmission enhanced achievable throughput, mitigated node and network energy consumption than the conventional method. Although, the design of a common control channel with a dedicated unlicensed ISM band is subjected to intruder attacks, which affects the output of the network.

Ji et al. [13] proposed a spectrum aware Semi Structure Routing (SSR), which incorporates power control for the CR networks. By using routing and forwarding zones, the SSR can effectively utilize the local real-time spectrum dynamics and it can guarantee the global routing. The proposed SSR method attained energy efficiency by completing each data transmission with allowed low power of the network. The number of the Primary Users (PU) has some impact on energy consumption analysis of the SSR scheme. Although the proposed method suffered by high latency performance.

Banerjee et al. [14] proposed joint Power Allocation (PA) and route selection for outage minimization in the multi-hop CR networks with energy harvesting. Radio frequency energy harvesting cooperation transmission was considered in this work, it operates in time switching mode. The relay helps to data transmission between the source and destination for energy consumption. The Global Minimum Point (GMP) of the secondary outage probability showed through convexity of the optimization function. The main disadvantage of Dijkstra's algorithm is the fact that it does a blind search there by consuming a lot of time waste of necessary resources.

Yang et al. [15] investigated a global optimization based on routing protocol for improving service quality in CR enabled advanced 


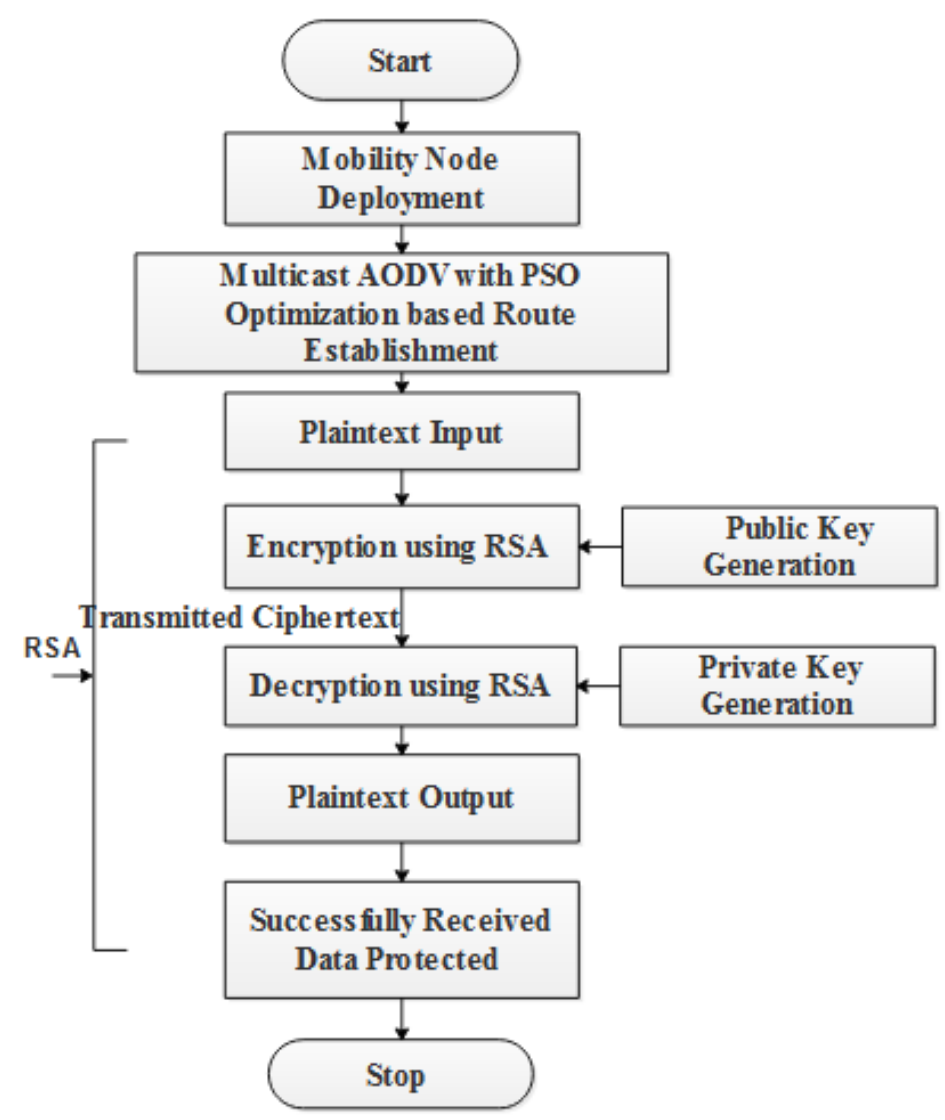

Figure. 1 Flowchart of the MAODV-PSO- RSA method

metering infrastructure networks. This paper proposed a new RP for lossy network and low power based routing protocol. This proposed protocol uses an optimization technique to find the best route in the entire network. In addition, the proposed MAODV routing protocol explicitly protects PUs and meets the utility necessities of the secondary network. But, some nodes affected by severe unreliability problems and higher packet loss rates because of the selection of the suboptimal paths with more unreliable links.

An efficient MAODV-PSO-RSA method is implemented for improving $\mathrm{CR}$ network performance and to overcome the above-mentioned problems.

\section{MAODV-PSO- RSA methodology}

Multicast is a communication method that allows one source node to transmit information to multiple destination nodes at one time. The multicast routing requires to detect a multicast between a source node and a group of destination nodes. This research represents a way to enhance the PDR, delay and energy consumption of the system by using backup paths. To select the best path among backup paths the PSO algorithm is applied and it also helps to find the best fitness value for further transmission during route repair process. The flowchart of the MAODV-PSO- RSA method is represented in Fig. 1. Initially, the sensor nodes are randomly organized in the region of the network for gathering the information from neighbor nodes. Hence, a number of nodes are varied by setting fixed nodes in the Cognitive ad-hoc networks. The reliable PSO optimization with MAODV routing protocol technique is used for best route selection in the Cognitive ad-hoc networks. Finally, the RSA is used to protect the transmitted data. The process is repeated until all the data packets received to the destination nodes in the Cognitive ad-hoc networks. A brief evaluation of the proposed MAODV- PSORSA method is described below.

\subsection{Multicast ad-hoc on-demand distance vector}

The proposed MAODV protocol structure is shown in Fig. 2, which is utilized to select the best route in the $\mathrm{CR}$ network. The principle of the MAODV routing protocol algorithm is briefly explained as follows. The multicast routing distributes the information to a group of the destinations, simultaneously. The multicast routing 


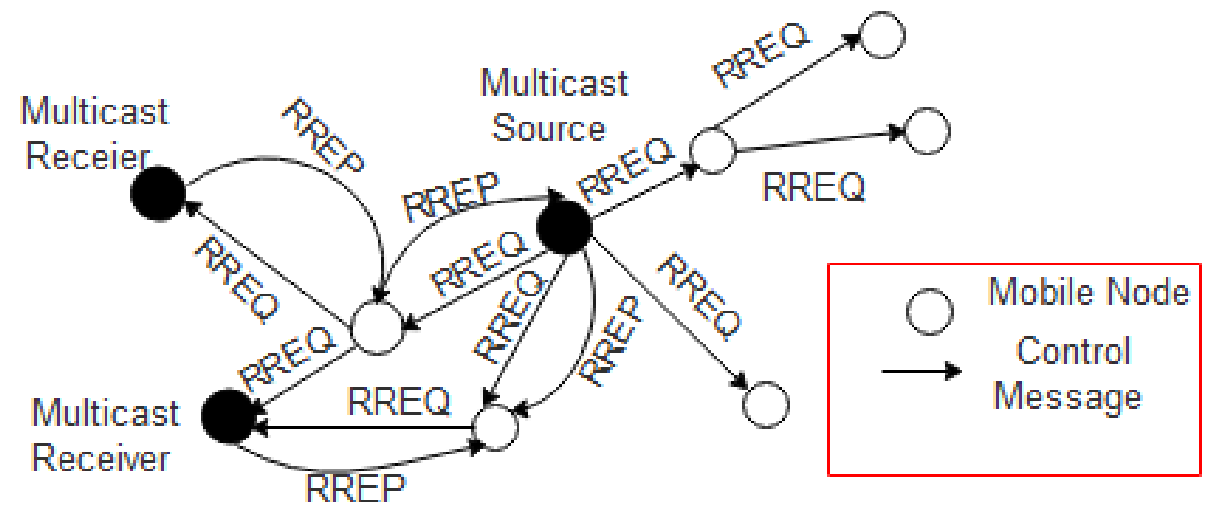

Figure. 2 Proposed MAODV protocol structure

protocol can be classified into 2 types such as Meshbased routing protocol and tree-based routing protocol. The mesh-based routing protocols use many routes to reach a destination, which is considered for this research. The tree-based protocols maintain only one path to reach destination [16]. Single route abstraction needs a source node to establish a new route discovery process when a link failure is encountered in the present primary route. How to achieve transmission in energy efficient way in CRS networks is significant since sensor nodes in CRS networks are generally battery powered [17]. So, this research selected the MAODV for routing process in the CR network. The multicast protocol is the main key approach of the group team applications, which has significantly reduced the network load when packets require to be transmitted to a group of nodes. The multicast protocol must assure the performance requirements such as timeliness, efficiency, adaptable to the dynamic change of network topology, and minimizing routing overhead. The multicast is a communication scheme for group information source using single source address to send data to host with the similar group address. The MAODV performs based on multicast mesh adopting Broadcast Routing Discovery Mechanism (BRDM) to search multicast routing, it sends data packets to each and every group node from the data source.

The proposed MAODV use Route Request (RREQ) and Route Reply (RREP), which already exist in AODV. If RREQ is not a joint request, updated routing path can respond directly at any node. Since the PDR is an important computation reliability for MAODV, the source node retransmits the similar packet again, if the data packet is not delivered properly. The energy consumption and PDR significantly improved along with end-end delay by using the MAODV algorithm. After the routing process, the PSO algorithm is applied to the routes for route optimization that improves the energy consumption of the CR network. The principle of the route optimization PSO algorithm is briefly explained below.

\subsection{PSO algorithm based route optimization}

The bio-inspired PSO algorithm have been successfully applied in a spectrum overlay context [18]. To overcome the non-linear continuous optimization issues, the stochastic optimization method based on swarm movement and intelligence is used. The PSO inspired by the sociological behavior of the bird flocking to achieve optimal results. The route is considered as a particle in this research work. The PSO contains a swarm of a predefined size of the particles. The flowchart of the proposed PSO is shown in Fig. 3.

In PSO algorithm, each particle provides a complete solution for the multidimensional optimization issue. The dimension $D$ of all the particles is equal. The particle $P_{i}, 1 \leq i \leq N_{P}$ has position to $X_{i d}, 1 \leq d \leq D$ and velocity $V_{i d}$ in $d^{t h}$ dimension of the hyperspace. This research adopts the notation for denoting the $i^{\text {th }}$ particle $P_{i}$ of population in Eq. (1).

$$
P_{i}=\left[X_{i, 1}, X_{i, 2}, X_{i, 3}, \ldots \ldots . X_{i, D}\right]
$$

Every particle computed by a fitness function to judge the better solution of the problem. To reach the best position, the particle $P_{i}$ follows its own best personal best $\left(P_{\text {besti }}\right)$, and global best $\left(G_{\text {best }}\right)$ to obtain its own position and velocity. In every iteration, its velocity $V_{i d}$ and position $X_{i d}$ in $d^{\text {th }}$ dimension is obtained by using the following Eqs. (2) and (3). 


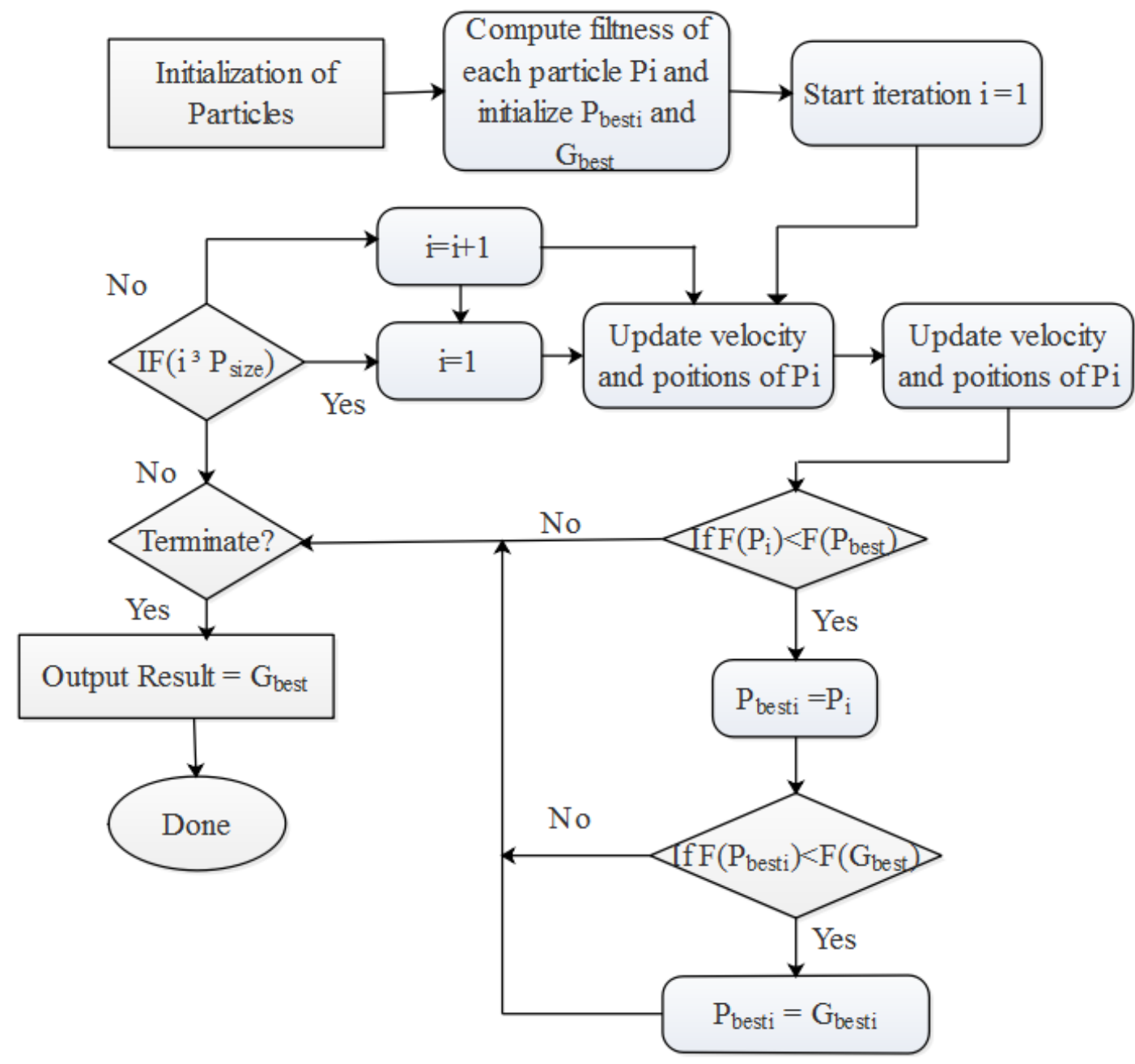

Figure. 3 Flowchart of proposed PSO

$$
\begin{aligned}
& \quad V_{i, d}(t)=w \times V_{i, d}(t-1)+c_{1} \times r_{1} \times \\
& \left(X_{\text {pbest }_{i, d}}-X_{i, d}(t-1)\right)+c_{2} \times r_{2} \times\left(X_{\text {gbest }_{d}}-\right. \\
& \left.X_{i, d}(t-1)\right) \\
& \quad X_{i, d}(t)=X_{i, d}(t-1)+V_{i, d}(t)
\end{aligned}
$$

Here, $w$ represents internal weight, $c_{1}$ and $c_{2}$ are two non-negative constants which are known as acceleration factor, $r_{1}$ and $r_{2}$ are 2 different uniform distributed random numbers in the network range. The update procedure is iteratively repeated until either acceptable $G_{\text {best }}$ is achieved. After the route optimization process, the security algorithm is applied on the optimized route. It is briefly described as follows.

\subsection{Rivest Shamir Adleman (RSA) security algorithm}

In this research, the RSA algorithm is used for securing sensitive data and secure data transmission, because RSA provides reliable encryption than other encryption standards. The RSA consists of four steps such as the generation of the key, distribution of the key, data encryption and data decryption. The public key will be known to all, but the private key is generated during the data encryption will be sent to the receiver node and a reasonable amount of time will be allotted to decrypt the data.

Input: Generate or choose large random prime numbers.

Output: Public Key $(n, e)$ and private key $(d)$.

1. Two different prime numbers such as $p$ and $q$ is generated.

2. Compute the modulus $n=p \times q$.

3. Compute the $\varphi(n)=(p-1) \times(q-1)$.

4. Choose for public exponent an integer $e$ such that $1<e<\varphi(n)$ and $\operatorname{gcd}(\varphi(n), e)=1$. 
5. Compute the private exponent $d=$ $e^{-1} \bmod \varphi(n)$ (employing the extended euclidean algorithm).

6. Public key $=(e, n)$.

7. Private $\operatorname{key}(d)$.

The Eq. (4) is written as Eq. (5) by interchanging $d$ and $e$ values.

$$
\begin{aligned}
& \left(m^{e}\right)^{d} \equiv m(\bmod n) \\
& \left(m^{d}\right)^{e} \equiv m(\bmod n)
\end{aligned}
$$

The sender $A$ can send encryption message to the $B$ without requiring prior exchange of secret keys. The data $A$ transmits through public key $(n, e)$ to $\mathrm{B}$ on a route, which is reliable. For encryption, it consider an integer $m$ that lies between $(0, n)$ such that $g c d$ of $\{m, n\}$ must be equal to one. From $m$ and $n$ the cipher text $c$ calculated, which shows in Eq. (6). The data is successfully transferred using RSA encryption algorithm. During $B$ sends the data to $A$ in decryption process the $\mathrm{s}, m$ is recovered from $C$ employing the private key exponential $d$ by Eq. (7).

$$
\begin{aligned}
& c=m^{e}(\bmod n) \\
& c \equiv\left(m^{e}\right)^{d} \equiv m(\bmod n)
\end{aligned}
$$

The RSA algorithm is a common public key cryptography for data encryption and decryption process. The powerful encryption and optimized key management scheme always help to achieve authentication and integrity of data and mitigate the overheads of a network system. In this algorithm, the key length is directly proportional to the security and inversely proportional of network performances. Hence, hacking time is reduced, which represent that the time available for hackers has been reduced. By this way, the data packets are securely sent source to destination. The simulation results of the MAODV-PSO-RSA method is described in the following section.

\section{Result and discussion}

This section briefly explained the experimental result of MAODV-PSO-RSA method. The MAODV-PSO-RSA algorithm was done in an NS2 software tool. The performance of the MAODVPSO-RSA is analyzed in terms of energy, PDR, end to end delay and packet loss. These performance parameters are taken by varying the number of
Table 1. Simulation parameters

\begin{tabular}{|c|c|}
\hline Parameters & Value/Types \\
\hline Routing Protocol & MAODV \\
\hline Route Optimization & PSO \\
\hline Security Algorithm & RSA \\
\hline Key Management & ID-SKMS \\
\hline Simulator Used & NS2 \\
\hline Simulation start time & $0.0000000001 \mathrm{~ms}$ \\
\hline Simulation end time & $50.000000000 \mathrm{~ms}$ \\
\hline Packet size & 1024 bits \\
\hline Number of mobile nodes & $20,40,60,80$ and 100 \\
\hline Antenna Model & Omni Antenna \\
\hline Minimum Speed & $28 \mathrm{~ms}$ \\
\hline Network Interface Type & Wireless \\
\hline MAC Type & MAC/802_11 \\
\hline
\end{tabular}

nodes (static/fixed nodes) and by a varying number of rates. The process repeats until entire data packets received to the destination nodes in the CR networks. The research work considered 100 nodes that are randomly placed in CR network region. The User Datagram Protocol (UDP) is used as a data agent. Table 1 shows the simulation parameters of proposed MAODV-PSO-RSA method.

In this research selected to make a comparison with BIRP [19] and IFLIP [20]. The proposed method is implemented to share the routing and data to all the nodes around the network. Here, the performance proposed and existing methods are analysed for 100 nodes. For this work, C, C++ and Tool Command Language (TCL) are selected to write the simulation code and the NS2 simulator is utilized for the simulation.

\subsection{Performance metrics}

Energy Consumption: The value of the energy consumption is considered as joules. The energy consumed on sleep, idle, transmit and receive with respect to total energy consumed, which is expressed in Eq. (8).

Energy consumption $=$

Current value - Initial energy value

End-to-end delay: The end to end delay defines to time taken by a packet to be transmitted across a network from the transmitter to the receiver. The performance of delay is measured in Sec. The end-to-end delay is expressed in Eq. (9).

End to End Delay $=$ Packet Recived Time Packet Transmited Time 
Packet Delivery Ratio: The PDR is the total percentage of the packets successfully received at the destination. The formula of PDR is expressed in Eq. (10).

$$
P D R=\frac{\text { Total number of packets received }}{\text { Total number of packets sent }}
$$

Packet Drop: The packet drop is the difference of both sending packets and receiving packets, which is known as the packet drop, which is expressed in Eq. (11).

\section{Packet Drop = Total number of packets sends-packet received} Total number of simulation

\subsection{Performance analysis}

Energy competence metrics are significantly utilized in various ways for different purpose, but it is widely used in comparing the efficiency performance and the energy consumption of various systems of same level. Performance of energy consumption for existing BIRP [19], IFLIP [20] method and MAODV-PSO-RSA method is shown in Fig. 4. In this research, the proposed routing protocol implemented two different types of schemes for less energy consumption and security on CR networks such as MAODV with PSO algorithm and AODV without PSO algorithm. The proposed PSO based MAODV routing protocol implemented for obtaining better optimal stable route way and it consumes less energy compared to other routes. In BIRP [19], the M/G/1 queuing model is used to avoid congestion in CRN that increases the overall network performance, but in some specific conditions, it failed to avoid congestion in large length queue in CRN. In IFLIP [20], the proposed routing protocol selects a short path without checking its quality and this leads to route failure problem in output. Due to the wrong selection of route, the enormous amount of energy is wasted. The MAODV-PSO-RSA algorithm obtained less energy consumption compared to existing methods such as BIRP [19] and IFLIP [20] because the PSO algorithm is used in the proposed routing protocol for best route selection, which is shown in Fig. 4. This research presented an energy consumption framework using MAODV-PSO-RSA for a given quality of the service requirement depends on the channel and radio capabilities.

Fig. 5 shows the performance of delay for existing BIRP [19] and IFLIP [20] and MAODVPSO-RSA method. The proposed MAODV-PSORSA algorithm attained less delay in data packet to reach destination. A proposed routing algorithm can be used for multi-channel CR network due to its less transmission delay. The PSO algorithm is used to detect the smallest transmission delay route of given packets on each link. The experimental simulation result showed that the proposed algorithm obtained less end-to-end delay for data packet transmission compared to existing methods BIRP [19], IFLIP [20].

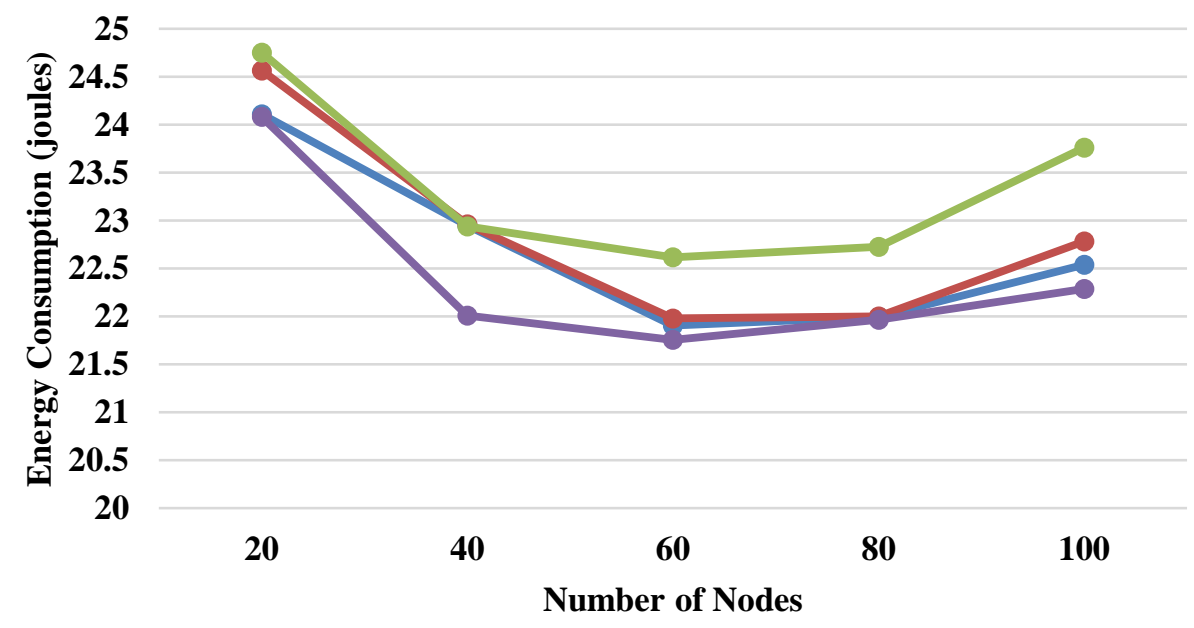

$\longrightarrow$ BIRP [19] $\rightarrow$ IFLIP [20] -MAODV $\rightarrow$ MAODV-PSO-RSA

Figure. 4 Performance of energy consumption for existing and MAODV-PSO-RSA algorithm 


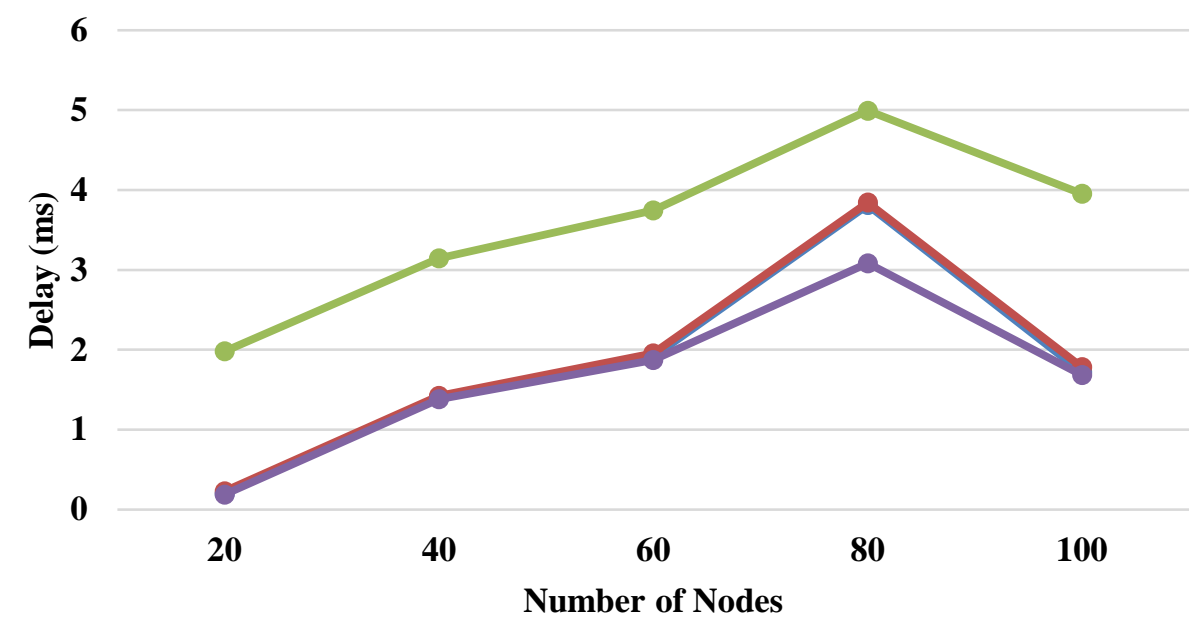

$\multimap$ BIRP [19] $\multimap$ IFLIP [20] $\multimap$ MAODV $\multimap$ MAODV-PSO-RSA

Figure. 5 Performance of delay for existing and MAODV-PSO-RSA algorithm
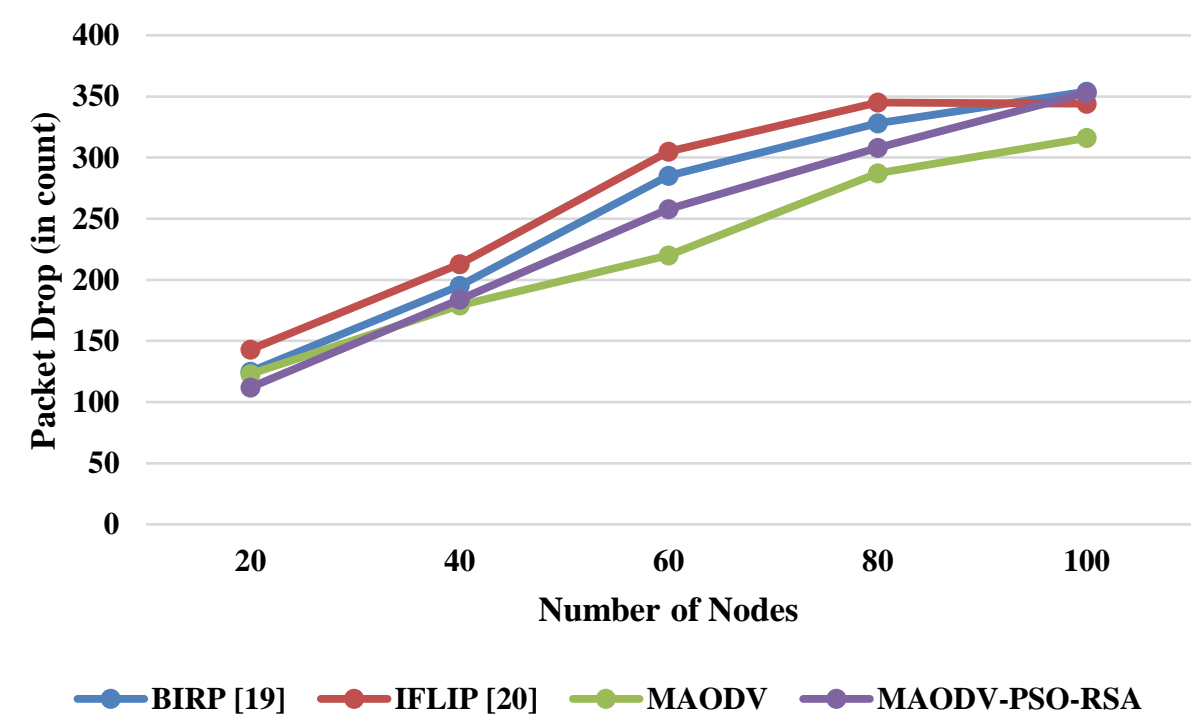

Figure. 6 Performance of packet drop for existing and MAODV-PSO-RSA algorithm

The performance of packet drop for existing BIRP [19] and IFLIP [20] and MAODV-PSO-RSA method is shown in Fig. 6. The proposed MAODVPSO-RSA is achieved less packet drops compared to existing BIRP [19] and IFLIP [20]. The performance of the packet drop is analyzed based on a number of the packets sent on the CR network that fail to reach their destination node. In the CR network, the packet drop is the main issue because of the high speed of traffic being identified in the network. But, the MAODV-PSO-RSA algorithm efficiently obtained less packet drop than BIRP [19] and IFLIP [20].

The performance of PDR for BIRP [19] and IFLIP [20] and MAODV-PSO-RSA is shown in Fig. 7. The proposed algorithm obtained high level of packet delivery ratio compared to the BIRP [19] and IFLIP [20]. The number of dropped packets is not taken into retransmissions and it creates the number of transmitted packets is equal to a total number of received packets and numbers dropped packets. The proposed method never fail to send the data packets, in congestion region it tries to sends the data packet in some other route to reach the destination with increasing the PDR.

This proposed method investigated multiple channels and determined the optimal transmission duration and energy consumption using an optimized routing protocol mechanism. In this research, the route maintenance is reconstructed automatically by using route error and route recovery messages. It is compared with the proactive routing protocol because proposed MAODV can create routing table when the demands 


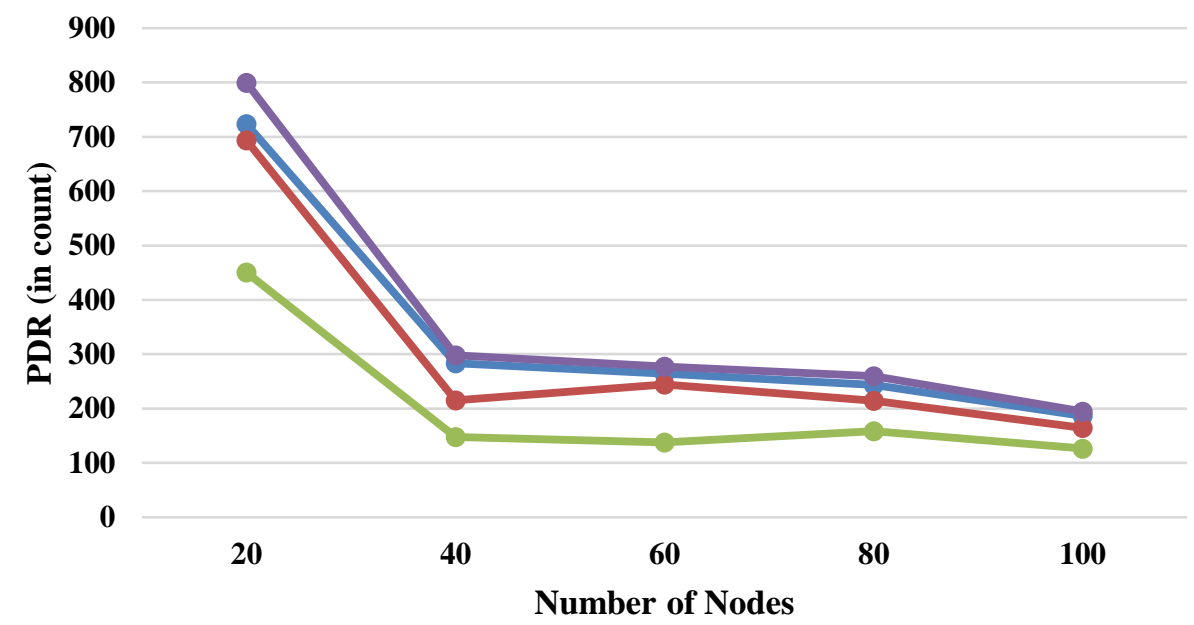

$\longrightarrow$ BIRP [19] $\longrightarrow$ IFLIP [20] $\longrightarrow$ MAODV $\longrightarrow$ MAODV-PSO-RSA

Figure. 7 Performance of PDR for existing and MAODV-PSO-RSA algorithm

occur. Finally, it concluded that PSO based MAODV routing protocol performed better in the CR network by analyzing performance parameters in terms of energy consumption and delay.

\section{Conclusion}

Reducing energy consumption and maintaining security is a major challenge task in CR networks. This research work aimed to reduce energy consumption in CRN by proposing effective routing and bio-inspired protocol. The proposed routing protocol selects the optimal routes in multiple routes for transferring the required data to the destination. The proposed protocol checks the quality of the routes before sending data, because sending through low quality routes may cause retransmission, congestion and waste of energy. This research work used PSO based multicast AODV to select the optimal route with less energy consumption. Finally, the transmitted data are secured by using RSA, because RSA provides reliable encryption than other encryption standards. The NS2 simulator is utilized to measure the performance of the proposed protocol against the existing protocols with benchmark performance metrics. The experimental outcomes show that the proposed method outperforms the existing methods in terms of reducing energy consumption, security and end-toend delay. In future work, the reliable routing protocol with efficient optimization techniques will be used to reduce energy consumption in CR ad-hoc networks like Hybrid invasive weed optimization or genetic algorithm, etc.

\section{References}

[1] H.N. Abdullah and H.S. Abed, "Improvement of energy consumption in cognitive radio by reducing the number of sensed samples", In: Proc. of Al-Sadeq International Conference on Multidisciplinary in IT and Communication Science and Applications (AIC-MITCSA), pp. 16, 2016.

[2] Z. Che-aron, A.H. Abdalla, K. Abdullah, W.H. Hassan, and M.A. Rahman, "A robust ondemand routing protocol for cognitive radio ad hoc networks", In: Proc. of Mobile and Wireless Technology, Springer, pp. 33-43, 2015.

[3] N. Mansoor, A.K.M. Muzahidul Islam, M. Zareei, and C. Vargas-Rosales, "RARE: A Spectrum Aware Cross-Layer MAC Protocol for Cognitive Radio Ad-Hoc Networks", IEEE Access, 6, pp. 22210-22227, 2018.

[4] E.F. Orumwense, T.J. Afullo, and V.M. Srivastava, "Secondary user energy consumption in cognitive radio networks", In: Proc. of AFRICON, pp. 1-5, 2015.

[5] N. Priya and B.R. Jeetha, "Energy Efficient Spectrum Sensing Routing Protocol (EESSRP) in Cognitive Radio Adhoc Network", In: Proc. of Al-Sadeq International Conference on Multidisciplinary in IT and Communication Science and Applications (AIC-MITCSA) IRAQ, pp. 9-10, 2016.

[6] A. Guirguis, M. Karmoose, K. Habak, M. ElNainay, and M. Youssef, "Cooperation-based multi-hop routing protocol for cognitive radio 
networks", Journal of Network and Computer Applications, Vol.110, pp.27-42, 2018.

[7] K. Singh and S. Moh, "Routing protocols in cognitive radio ad hoc networks: A comprehensive review", Journal of Network and Computer Applications, Vol.72, pp.28-37, 2016.

[8] K. R. Chowdhury and I. F. Akyildiz, "CRP: A Routing Protocol for Cognitive Radio Ad Hoc Networks", IEEE Journal of Selected Areas in Communications, Vol.29, No.4, pp.794-804, 2011.

[9] A.C. Talay and D.T. Altilar, "ROPCORN: Routing protocol for cognitive radio ad hoc networks", In: Proc. of International Conference on Ultra-Modern Telecommunications \& Workshops (ICUMT'09), pp. 1-6, 2009.

[10] J. Wang, H. Yue, L. Hai, and Y. Fang, "Spectrum-aware anypath routing in multi-hop cognitive radio networks", IEEE Transactions on Mobile Computing, Vol.16, No.4, pp.11761187, 2017.

[11] Y. Wang, G. Zheng, H. Ma, Y. Li, and J. Li, "A Joint Channel Selection and Routing Protocol for Cognitive Radio Network", Wireless Communications and Mobile Computing, 2018.

[12] S. Anamalamudi, A.R. Sangi, M. Alkatheiri, and A.M. Ahmed, "AODV routing protocol for Cognitive radio access based Internet of Things (IoT)", Future Generation Computer Systems, Vol.83, pp.228-238, 2018.

[13] S. Ji, M. Yan, R. Beyah, and Z. Cai, "Semistructure routing and analytical frameworks for cognitive radio networks", IEEE Transactions on Mobile Computing, Vol.15, No.4, pp.9961008, 2016.

[14] A.Banerjee, A. Paul, and S.P. Maity, "Joint power allocation and route selection for outage minimization in multihop cognitive radio networks with energy harvesting", IEEE Transactions on Cognitive Communications and Networking, Vol.4, No.1, pp.82-92, 2018.

[15] Z. Yang, S. Ping, A. Aijaz, and A.H. Aghvami, "A global optimization-based routing protocol for cognitive-radio-enabled smart grid AMI networks", IEEE Systems Journal, Vol.12, No.1, pp.1015-1023, 2018.

[16] S.R. Gowda and P.S. Hiremath, "Review of security approaches in routing protocol in mobile adhoc network", International Journal of Computer Science Issues, Vol.10, No.1, pp.242, 2013.

[17] H. Bin, M. Zeng, Q. Guo, H. Jiang, Q. Zhang and L. Feng, "Energy efficient sensing and transmission for multi hop relay cognitive radio sensor network", China Communications, Vol.15, No.9, pp. 106-117, 2018.

[18] D.M. Chander, "PSO Based Route Optimization Technique to Enhance Network Lifetime", International Journal of Advanced Research in Computer Science and Software Engineering, Vol.6, No.7, 2016.

[19] R. Jaganathan and V. Ramasamy, "Performance Modeling of Bio-Inspired Routing Protocols in Cognitive Radio AdHoc Network to Reduce End-to-End Delay", International Journal of Intelligent Engineering and Systems, Vol.12, No.1, pp.221-231, 2019.

[20] J. Ramkumar and R. Vadivel, "Improved Frog Leap Inspired Protocol (IFLIP) - for Routing in Cognitive Radio Ad Hoc Networks (CRAHN)", World Journal of Engineering, Vol.15, No.2, pp.306-311, 2018. 